\title{
Vergütungsstrukturen von Versorgungsnetzwerken
}

\author{
Reembursing Health-Care Service Provider Networks
}

Autoren

Institute

\author{
A. Binder ${ }^{1}$, G. E. Braun
}

${ }^{1}$ Fakultät für Wirtschafts- und Organisationswissenschaften, Universität der Bundeswehr München, Neubiberg ${ }^{2}$ Institut für Betriebswirtschaftslehre des öffentlichen Bereichs und Gesundheitswesens, Universität der Bundeswehr München, Neubigerg

\section{Schlüsselwörter \\ - Versorgungsforschung \\ - Gesundheitsnetzwerke \\ - Vergütung \\ - virtuelles Budget \\ - Capitation}

\section{Key words}

- health-care research

health-care service provider networks

- reimbursement

- virtual budgets

- capitation

\section{Zusammenfassung \\ $\nabla$}

Gesundheitsnetzwerke als innovative Versorgungsformen gelten als wichtiges Instrument, um die vielfach beklagte Fragmentierung und sektorale Gliederung des Gesundheitswesens zu überwinden. Im ersten Teil des Beitrags werden Gesundheitsnetzwerke in das Feld Versorgungsforschung eingeordnet. Dazu werden das system-theoretische Modell sowie grundlegende Funktionen der Versorgungsforschung herangezogen. Auch werden bereits etablierte Teilgebiete der Versorgungsforschung mit besonderem Bezug zu Gesundheitsnetzwerken benannt. Im zweiten Teil werden innovative Gestaltungsoptionen für die Vergütung von Gesundheitsnetzwerken als Ergebnisse einer netzwerkbezogenen Versorgungsforschung vorgestellt. Den Ausgangspunkt bilden dabei Vergütungsstrukturen für virtuelle Budgets, wie sie derzeit zum Teil im Bereich der integrierten Versorgung nach $\S \S 140$ a ff. SGB V umgesetzt werden. Aus der Beschreibung und Bewertung dieses Modells ergibt sich als konsequente Weiterentwicklung eine Vergütung durch reale Budgets (Capitation) eine Vergütungsform, die z.B. durch den SVRGesundheit wiederholt gefordert aber in der Praxis bisher kaum umgesetzt wurde. In einem weiteren Entwicklungsschritt wird schließlich eine Vergütung von Netzwerken direkt aus dem Gesundheitsfonds diskutiert. Dabei zeigen sich wesentliche Vorteile des Modells aber auch Herausforderungen. Bei der Entwicklung der jeweiligen Vergütungsmodelle werden teilweise Vorbilder aus den USA einbezogen.

Gesundheitsnetzwerke als innovative Versorgungsformen gelten als wichtiges Instrument, um die vielfach beklagte Fragmentierung und sektorale Gliederung des Gesundheitswesens zu überwinden. Ihr Ziel ist es, durch die Integration der Leistungserstellung und -finanzierung die

\section{Abstract \\ $\nabla$}

Health-care service provider networks are regarded as an important instrument to overcome the widely criticised fragmentation and sectoral partition of the German health-care system. The first part of this paper incorporates health-care service provider networks in the field of health-care research. The system theoretical model and basic functions of health-care research are used for this purpose. Furthermore already established areas of health-care research with strong relations to health-care service provider networks are listed. The second part of this paper introduces some innovative options for reimbursing health-care service provider networks which can be regarded as some results of network-oriented health-care research. The origins are virtual budgets currently used in part to reimburse integrated care according to $\S \S 140 \mathrm{a}$ ff. SGB V. Describing and evaluating this model leads to real budgets (capitation) - a reimbursement scheme repeatedly demanded by SVR-Gesundheit (German governmental health-care advisory board), for example, however barely implemented. As a final step a direct reimbursement of networks by the German sickness fund is discussed. Advantages and challenges are shown. The development of the different reimbursement schemes is partially based on models from the USA.
Korrespondenzadresse

Dr. Andreas Binder

Chausseestraße 104

10115 Berlin

andreas.binder.75@web.de

\section{License terms}

DOI http://dx.doi.org/

Online-Publikation: 27.1.2015

Gesundheitswesen 2015;

(c) Georg Thieme Verlag KC

Stuttgart $\cdot$ New York

(๑) $\Theta \Theta$
Qualität der Versorgung zu verbessern und gleichzeitig ihre Wirtschaftlichkeit zu steigern $[1,2]$. Sie können der Umsetzung innovativer Handlungsformen des SGB V dienen wie z.B. der integrierten Versorgung nach $\S \S 140 a$ ff. SGB V [3]. Als sog. Praxisnetze in der ambulanten Medi- 
Tab. 1 Funktionen der Versorgungsforschung, vgl. zu den Inhalten [7, 8].

\begin{tabular}{|c|c|c|}
\hline Funktion & Fragestellung & Beschreibung \\
\hline Beschreibung & Welche Versorgungssituation liegt vor? & naturalistisch, deskriptiv, Ist-Situation \\
\hline Erklärung & Wie ist die vorliegende Versorgungssituation kausal begründet? & $\begin{array}{l}\text { naturalistisch, analytisch, Abklärung von Einflussfaktoren, } \\
\text { Bedingungen, Ursachen, Wirkungszusammenhänge }\end{array}$ \\
\hline Gestaltung & $\begin{array}{l}\text { Wie lässt sich die analysierte Versorgungssituation durch gezielte Interven- } \\
\text { tionen auf Basis versorgungswissenschaftlicher Erkenntnisse verbessern? }\end{array}$ & $\begin{array}{l}\text { interventionell, empirie- und theoriebasierte Fach- und } \\
\text { Politikberatung }\end{array}$ \\
\hline Begleitevaluation & Welche Probleme treten bei der Umsetzung der entwickelten Maßnahmen auf? & formativ, naturalistisch, interventionell \\
\hline Ergebnisevaluation & Welche Wirksamkeit erzielt die Intervention unter Alltagsbedingungen? & summativ, Orientierung an der Effectiveness \\
\hline
\end{tabular}

zin sind sie seit kurzem nach $§ 87 b$ SGB V als förderungswürdige Organisationsform anerkannt. In diesem Beitrag werden Gesundheitsnetzwerke aus der Perspektive der Versorgungsforschung behandelt und neue Gestaltungsoptionen für anreizkompatible Vergütungsstrukturen solcher Netze entwickelt.

\section{Versorgungsforschung}

Der Wissenschaftliche Beirat bei der Bundesärztekammer definiert Versorgungsforschung als „die wissenschaftliche Untersuchung der Versorgung von Einzelnen und der Bevölkerung mit gesundheitsrelevanten Produkten und Dienstleistungen unter Alltagsbedingungen“ [4].

Pfaff dagegen versteht unter Versorgungsforschung „ein fachübergreifendes Forschungsgebiet, das die Kranken- und Gesundheitsversorgung und ihre Rahmenbedingungen beschreibt und kausal erklärt, zur Entwicklung wissenschaftlich fundierter Versorgungskonzepte beiträgt, die Umsetzung neuer Versorgungskonzepte begleitend erforscht und die Wirksamkeit von Versorgungsstrukturen und -prozessen unter Alltagsbedingungen evaluiert“" [5].

Als konzeptionellen Ausgangspunkt der Modellbildung im Bereich der Versorgungsforschung wird inzwischen verbreitet das systemtheoretische Input-Throughput-Output-OutcomeModell nach Pfaff genutzt [5].

Es ermöglicht zunächst durch den Einschluss unterschiedlichster Interventionen bzw. Versorgungsaktivitäten in die Black Box des Throughputs eine Reduktion der Komplexität und Heterogenität des Versorgungsgeschehens und erschließt so eine allgemeine Forschungsmethodik. Allerdings muss Versorgungsforschung über diesen Ansatz hinaus danach trachten, die Black Box des Throughputs aufzubrechen und die internen Wirkungszusammenhänge auch im Detail zu erforschen. Dies ist insbesondere für die Entwicklung und Empfehlung von differenzierten Gestaltungsoptionen von großer Bedeutung. Eine dezidiert ökonomische Perspektive eröffnet das Modell schließlich, wenn der Outcome in Relation zum Input gesetzt und so Effizienz gemessen wird [6].

In Bezug auf die methodischen Standards ist die Versorgungsforschung darum bemüht, ein möglichst hohes Niveau zu etablieren - nicht zuletzt, um ihre Ergebnisse gegenüber der klinischen Forschung zu behaupten [7]. Aufgrund Ihres Anspruchs, das Versorgungsgeschehen unter Alltagsbedingungen $\mathrm{zu}$ erforschen, sind die etablierten klinischen Standards der medizinischen Forschung, die in der Regel experimentelle Studiendesigns (RCT) vorsehen, aus praktischen oder auch ethischen Gründen nicht durchführbar, sodass auf Beobachtungsstudien und Quasi-Experimente zurückgegriffen werden muss. Dies ist nicht Ausdruck von Anspruchslosigkeit, Bequemlichkeit oder Willkür, sondern das angesichts des Anspruchs „unter Alltagsbedingungen“ maximal mögliche. Neugebauer, Pfaff und Schrappe unterscheiden 5
Funktionen bzw. Aufgaben der Versorgungsforschung, die in $\odot$ Tab. 1 dargestellt und näher beschrieben werden.

Im Folgenden werden Gesundheitsnetzwerke zunächst beschreibend charakterisiert. Im Anschluss werden dann ausgewählte Ergebnisse einer netzwerkbezogenen Versorgungsforschung präsentiert.

\section{Gesundheitsnetzwerke}

Nach Sydow stellt ein Unternehmungsnetzwerk „eine auf die Realisierung von Wettbewerbsvorteilen zielende Organisationsform ökonomischer Aktivitäten dar, die sich durch komplex-reziproke, eher kooperative denn kompetitive und relativ stabile Beziehungen zwischen rechtlich selbständigen, wirtschaftlich jedoch zumeist abhängigen Unternehmungen auszeichnet“ [8]. Im Bereich des Gesundheitswesens charakterisieren Braun und Güssow Netzwerke wie folgt: In Gesundheitsnetzen kooperieren unterschiedliche Dienstleistungsunternehmen des Gesundheitswesens. Typisch ist ein hoher Anteil von Freiberuflern bzw. Selbstständigen. Teilweise beteiligen sich auch (gesetzliche) Krankenversicherungen und andere Einrichtungen mit nicht erwerbswirtschaftlicher Zielsetzung. Gesundheitsnetze streben nach Überwindung des fragmentierten Handelns der Leistungsanbieter im Gesundheitswesen mithilfe einer zielgerichteten Koordination zum Wohl des Patienten als Hauptziel und gleichzeitiger Sicherstellung der Wettbewerbsfähigkeit der Netzmitglieder als Nebenziel. Insofern stellen Gesundheitsnetzwerke eine moderne Kooperationsform zwischen Leistungsanbietern im Gesundheitswesen dar. Sie bleiben rechtlich weitgehend selbstständig, geben aber ihre wirtschaftliche Selbstständigkeit partiell auf, um effektiver und effizienter zu handeln. Dennoch verfolgen Netzmitglieder zum Teil auch Eigeninteressen (z.B. Sicherstellung des eigenen Patientenstamms in Arztpraxen). Gesundheitsnetze können horizontale (z.B. in Praxisnetzen), vertikale (z.B. zusätzlich mit Krankenhäusern) und laterale (z.B. mit Wellness- oder Fitnesseinrichtungen) Kooperationsformen einschließen. Ferner können fokale Gesundheitsnetzwerke z. B. mit der Dominanz einer Krankenkasse oder eines Krankenhauses, einer (Groß-) Praxis, eines Gesundheits-, Facharzt-, bzw. Medizinischen Versorgungszentrums (MVZ) oder polyzentische (dezentrale) Netzwerke, z.B. in Praxisnetzwerken, unterschieden werden [9].

Zu den Teilgebieten der Versorgungsforschung mit besonderem Bezug zu Gesundheitsnetzwerken zählen die Gesundheitssystemforschung (auf der Mikro- und Mesoebene des Gesundheitssystems), die Erforschung komplexer Interventionen und die organisationsbezogene Versorgungsforschung. In Bezug auf das bereits angesprochene Input-Throughput-Output-Outcome-Modell der Versorgungsforschung von Pfaff sind Gesundheitsnetzwerke dem Throughput zuzuordnen, ๑ Tab. 2. 
Tab. 2 Gesundheitsnetzwerke im Input-Throughput-Output-Outcome-Modell (Parameter exemplarisch).

$\begin{array}{llll}\text { Input } & \text { Throughput } & \text { Output } & \text { Outcome } \\ \text { - Personal/Wissen } & - \text { Netzwerkorganisation } & - \text { Versorgungsleistung } & - \text { Lebensqualität } \\ \text { - Medizinische Akteure (Ärzte, Fachärzte, ggf. } & - \text { Leitlinien } & - \text { Behandlung/Therapie } & - \text { Mortalität } \\ \text { Krankenhäuser, ggf. Reha-Einrichtungen, ggf. } & \text { - Behandlungspfade } & - \text { Information und Beratung } & - \text { Prävalenz } \\ \text { Arzneimittelhersteller) } & \text { - Präventionsprogramme } & \text { - Patientensteuerung } & - \text { Inzidenz } \\ \text { - BWL-Know-how (Controlling, Marketing, } & \text { - Disease Management } & \text { - Surrogatparameter } & \text { - Medizinische indikationsbezogene } \\ \text { Gründungsmanagement und laufendes } & \text { - Case Management } & - \text { (z. B. HbA1c-Wert) } & \text { Outcome-Maße } \\ \text { Netzwerk-management) } & \text { - Primärarztprinzip } & \text { - Komplikationsraten } & \text { - Ökonomische Outcomes (Kosten, } \\ \text { - Patienten (Vorerkrankungen, Risikofaktoren) } & - \text { Vergütungsstrukturen/Anreize } & \text { - Wiederaufnahmeraten } & \text { Deckungsbeitrag) }\end{array}$

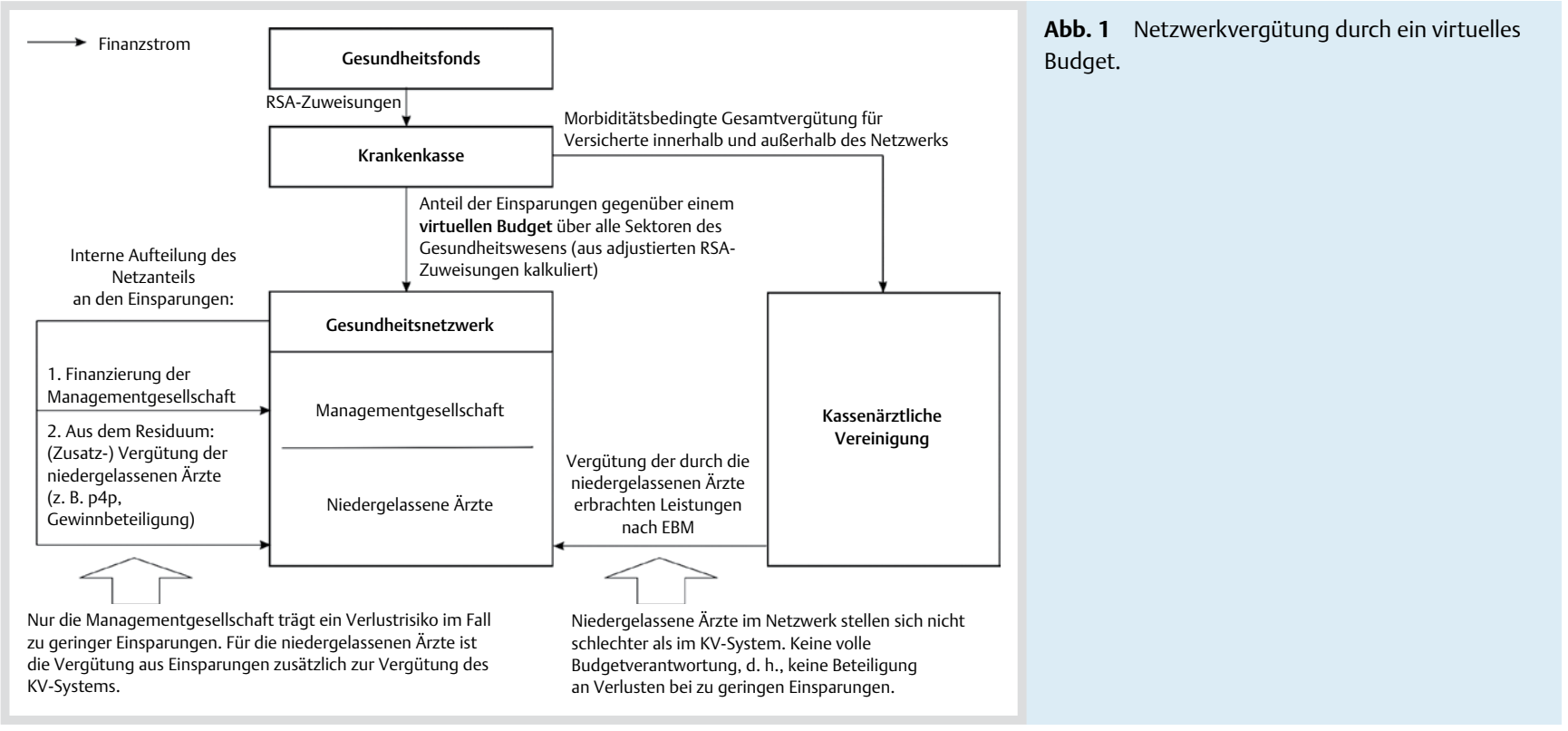

Die Integration der Leistungserstellung in Gesundheitsnetzen kann in 3 Dimensionen beschrieben werden [10]:

1. Die Integrationstiefe über die verschiedenen Sektoren entlang der Wertschöpfungskette im Gesundheitswesen

2. Die Indikationsbreite der angebotenen Versorgung

3. Das Ausmaß der Budgetverantwortung bzw. Risikoteilung aufgrund der gewählten Vergütungsformen.

Den Vergütungsformen für Netzwerke kommt eine Schlüsselfunktion zu, da sie wichtige Anreize setzen, die das Verhalten der Netzmitglieder bestimmen. Pauschale Vergütungsformen fördern die Angleichung der Interessen der Leistungserbringer an jene der Leistungsfinanzierer im Sinne der Prinzipal-AgentTheorie. Sektorübergreifende Budgets wirken nach dem Property-Rights-Ansatz Budgetegoismen zulasten der Versorgungsqualität in anderen Sektoren entgegen und fördern die Kooperation über die Sektorengrenzen hinweg. Qualitätsabhängige Vergütungskomponenten stärken die Versorgungsqualität im Sinne von Pay-for-Performance (p4p). Als aktuelle sozialrechtliche Handlungsformen zur Übernahme von Budgetverantwortung gelten die integrierte Versorgung nach $\S \S 140 \mathrm{a} f f . S G B V$, die besondere ambulante Versorgung nach $\S 73$ c SGB V, die hausarztzentrierte Versorgung nach $\S 73 \mathrm{~b}$ SGB V, die Strukturverträge nach $\S 73 a$ SGB V sowie die Modellvorhaben nach §§ 63-65 SGB V.

Nachfolgend werden innovative Möglichkeiten zur Vergütung von Gesundheitsnetzwerken in Deutschland präsentiert [11,12]. Dabei wird die Black Box des Throughputs von Gesundheitsnetzwerken aufgebrochen, um verschiedene Gestaltungskonzepte zu analysieren. Ergänzt wird die Perspektive der Gestal- tung dabei durch die beschreibende und erklärende Funktion der Versorgungsforschung ( $\bullet$ Tab. 1). Der Scherpunkt liegt dabei auf dem Bereich von Netzwerken niedergelassener Ärzte. Teile der diskutierten Gestaltungsoptionen werden im Sinne der Gesundheitssystemforschung anhand von Erfahrungen mit vergleichbaren Strukturen im Gesundheitssystem der USA gewonnen.

\section{Netzwerkvergütung durch virtuelle Budgets \\ $\nabla$}

\section{Grundlagen und Finanzströme}

Sog. Einsparverträge mit virtuellem Budget zielen auf die Erwirtschaftung von Einsparungen gegenüber einem sektorübergreifenden, prospektiv zwischen Netzwerk und Krankenkasse vereinbarten, virtuellen Budget [13-15].

Erwirtschaftete Einsparungen werden nach einem ex ante vertraglich festgelegten Verhältnis zwischen den Vertragspartnern geteilt. Die einzelnen niedergelassenen Vertragsärzte innerhalb des Netzwerks erhalten ihren Anteil meist in Form von Gewinnausschüttungen und leistungsabhängigen Prämien. Virtuell ist das Budget, weil die kooperierenden niedergelassenen Ärzte die von ihnen selbst erbrachten Leistungen wie in der Regelversorgung gegenüber der kassenärztlichen Vereinigung (KV) nach erweitertem Bewertungsmaßstab (EBM) abrechnen und ihr Honorar dafür aus der morbiditätsbedingten Gesamtvergütung erhalten [16]. Die Finanzbeziehungen zwischen den Akteuren einer populationsbezogenen Vollversorgung mit Einsparvertrag und virtuellem Budget werden in $\boldsymbol{\bullet}$ Abb. 1 dargestellt. 


\section{Quellen für Einsparungen}

Möglichkeiten zur Erwirtschaftung von Einsparungen durch ein Gesundheitsnetzwerk bestehen generell in einer verbesserten Organisation der intra- und intersektoralen Schnittstellen, in einer Verringerung der Morbidität durch Präventionsprogramme und in verbesserten Bedingungen beim Einkauf externer Produkte [17]. Letzteres kann auch zulasten von nicht beteiligten Sektoren gehen.

\section{Interne Budgetverteilung}

Die Möglichkeiten im Innenverhältnis andere Vergütungsstrukturen für die teilnehmenden Ärzte zu schaffen, beschränken sich bei einem virtuellen Budget auf die Verteilung möglicher Einsparungen. Da die Ärzte ihre Leistungen weiterhin über die KV abrechnen, wird der größte Teil ihres Einkommens durch deren Vergütungsstrukturen (EBM, Honorarverteilung, Budgets) bestimmt. Die Möglichkeit, an Einsparungen zu profitieren, ergänzt daher in Bezug auf die beteiligten niedergelassenen Ärzte nur die Anreize, die von den Vergütungsstrukturen der KV ausgehen.

Gesundheitsnetze mit virtuellem Budget existieren auch in den USA. Zu nennen sind insbesondere Einsparverträge zwischen sog. Accountable Care Organizations (ACOs) und Medicare, dem großen staatlichen Gesundheitsdienst für ältere Menschen [18]. ACOs werden im Gegensatz zu managed care-orientierten Versorgungs- und Versicherungsangeboten mit realem Budget (s. u.) Medicare-intern der Regelversorgung zugeordnet. [19-21].

\section{Budgetbereinigung}

Ein entscheidender Vorteil eines Einsparvertrags mit virtuellem Budget besteht darin, dass eine Bereinigung der morbiditätsbedingten Gesamtvergütung nicht notwendig ist, da die beteiligten niedergelassenen Ärzte die von ihnen erbrachten Leistungen weiterhin über die KV abrechnen [16]. Die Entwicklung und Umsetzung eines komplexen Bereinigungsverfahrens und damit verbunden mögliche Konflikte mit der KV können daher vermieden werden.

Unproblematisch ist bei einem virtuellen Budget auch der Fall, in dem Versicherte Leistungen von Ärzten außerhalb des Netzes in Anspruch nehmen, da gegen das virtuelle Budget alle im niedergelassenen Bereich erbrachten Leistungen gerechnet werden, unabhängig davon, ob der Leistungserbringer dem Netz angehört oder nicht.

\section{Verlustbeschränkungen}

Wenn keine Einsparungen erwirtschaftet werden oder das Budget sogar überschritten wird, erzielt die Managementgesellschaft keine Einnahmen, muss aber dennoch ihre laufenden Kosten (Personal, Netzinfrastruktur) decken, sodass Verluste entstehen, deren Aufteilung ebenfalls vertraglich zu bestimmen ist. Die teilnehmenden Ärzte erhalten in diesem Fall in der Regel keine Gewinnbeteiligung und auch keine leistungsabhängigen Vergütungsanteile ( $\mathrm{p} 4 \mathrm{p})$. Ihre selbst erbrachten Leistungen bekommen sie aber bei virtuellem Budget in voller Höhe durch die KV vergütet. Ärzte, die an der Managementgesellschaft als Gesellschafter beteiligt sind, haften darüber hinaus mit ihrer Einlage [17]. Das Risiko der Ärzte beschränkt sich daher auf die anteilige Haftung für Verluste. Sie übernehmen deshalb auch nur einen Teil des Versicherungsrisikos. Es kann somit nicht von voller Budgetverantwortung gesprochen werden und es findet nur eine begrenzte Interessenangleichung der Leistungserbringer an die der Leistungsfinanzierer statt. Aus diesem Grund erhalten die Krankenkassen für den Verlustfall in der Regel ein Sonderkündigungsrecht.

Eine Verlustbegrenzung für die teilnehmenden Ärzte ist jedoch nicht zwingend mit einem virtuellen Budget verbunden. Wenn das Netzwerk die Krankenkasse für Budgetüberschreitungen entschädigt, ist eine Risikoübernahme bis hin zur vollen Budgetverantwortung möglich. Medicare z.B. praktiziert dieses Modell in den USA gegenüber großen, am Markt etablierten ACOs [21]. Zusätzlich zu allgemeinen Verlustbegrenzungen werden in der Regel Rückversicherungsmodelle für Hochkostenfälle vereinbart.

\section{Risikoadjustierung}

Ohne Risikoadjustierung des Budgets entstehen mit der partiellen Risikoübernahme durch ein Gesundheitsnetzwerk Anreize zur Risikoselektion. Eine Risikoadjustierung des Budgets wird erreicht, indem es aus risikoadjustierten versichertenbezogenen Kopfpauschalen zusammengesetzt wird. Zu deren Kalkulation wird in der Praxis pragmatisch auf die risikoadjustierten Zuweisungen, die die Krankenkassen aus dem Gesundheitsfonds erhalten, zurückgegriffen. Da diese jedoch auf bundesweiten Durchschnittswerten beruhen, müssen sie an die Spezifika der jeweiligen regionalen Versorgungssituation angepasst werden [17]. Zur regionalen Adjustierung der morbiditätsadjustierten Kopfpauschalen wird derzeit die Morbiditätsdifferenz der Region zum Bundesdurchschnitt in einem Basisjahr vor Beginn der Tätigkeit des Netzes in der Region erfasst und über die Jahre fortgeschrieben. Die bundesweiten Durchschnittswerte werden dementsprechend angepasst.

Eine zusätzliche Maßnahme zur Vermeidung von Risikoselektion ergreift das Gesunde Kinzigtal: Die erwirtschafteten Einsparungen werden dort nicht in Bezug auf die im Netzwerk eingeschriebenen Versicherten berechnet, sondern in Bezug auf alle Versicherten der kooperierenden Krankenkassen in der Region. Für die Bestimmung der erwirtschafteten Einsparungen ist es daher egal, welche Versicherten der kooperierenden Krankenkassen eingeschrieben sind. Anreize, gute Risiken (Junge, Gesunde) einzuschreiben und schlechte Risiken (Alte, Kranke) in der Regelversorgung zu belassen, werden so vermieden.

\section{Netzwerkvergütung durch reale Budgets $\boldsymbol{\nabla}$}

\section{Grundlagen und Finanzströme}

Die Übernahme von Verantwortung für ein reales, ex ante zu Verfügung gestelltes und mit befreiender Wirkung gezahltes Budget impliziert ohne zusätzliche vertragliche Vereinbarungen volle Budgetverantwortung und wurde insbesondere durch den SVR-Gesundheit immer wieder angemahnt [1]. 2012 ist dem Unternehmen Gesundheit Oberpfalz Mitte (UGOM) erstmals der Abschluss eines entsprechenden Vertrags gelungen [14,22]. Vorbilder für eine Vergütung von Leistungserbringergemeinschaften durch risikoadjustierte Kopfpauschalen in Verbindung mit qualitätsorientierten Vergütungselementen existieren in Form der Medicare Advantage Plans in den USA [23]. Sie werden im Gegensatz zu den bereits erwähnten ACOs mit virtuellem Budget in einem eigenen, managed care-orientierten Part $C$ geführt $[24,25]$. Die Geldflüsse zwischen den Akteuren einer regionalen Vollversorgung mit voller Verantwortung für ein reales Budget werden in $\odot$ Abb. 2 dargestellt. 


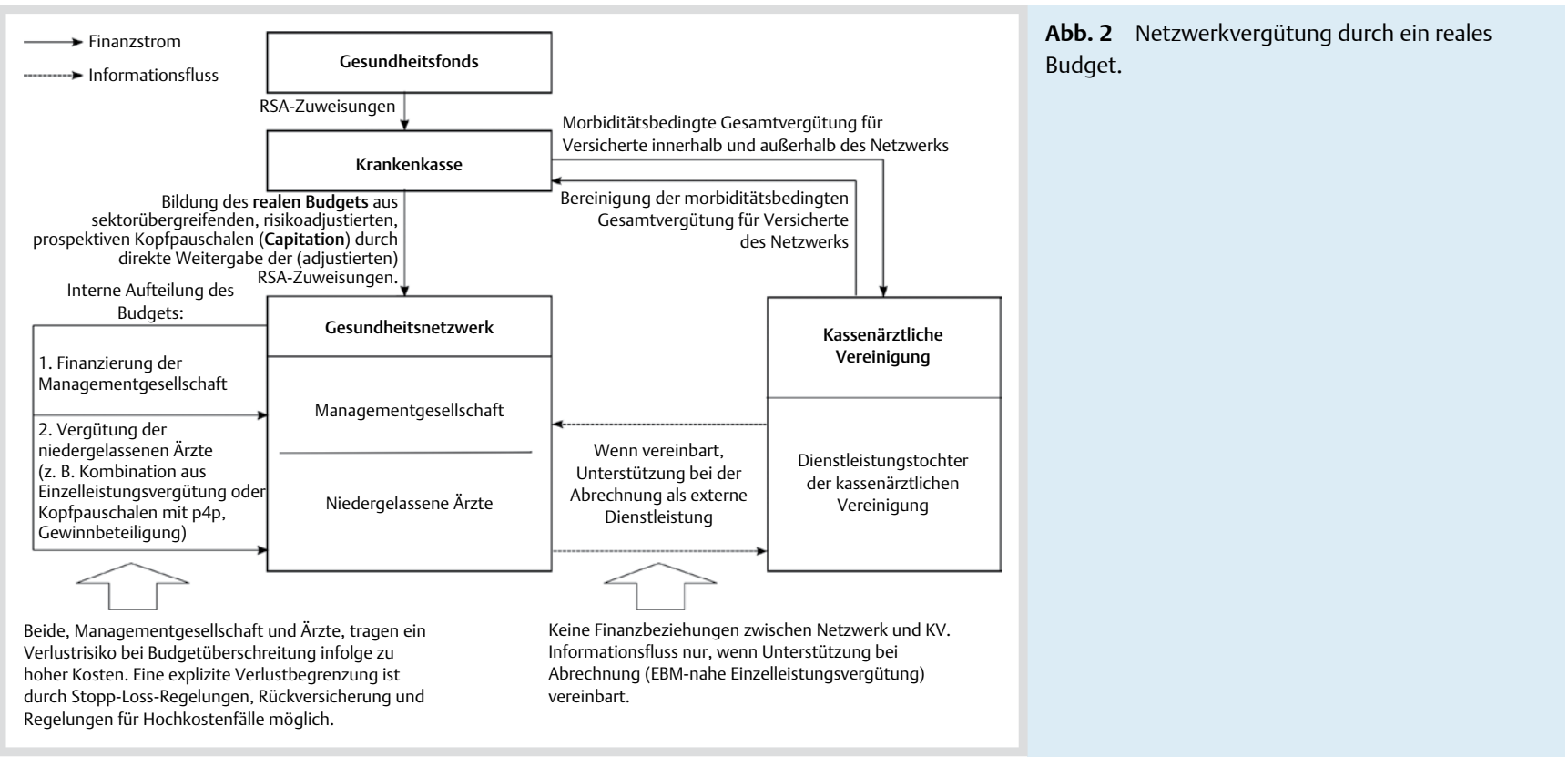

\section{Budgetkalkulation}

Die Kalkulation des realen Budgets kann analog zur Festlegung des virtuellen erfolgen, d.h. auf Basis der regional adjustierten Zuweisungen aus dem Risikostrukturausgleich [26]. Durch die höhere Risikobeteiligung im Vergleich zu einem virtuellen Budget mit Verlustbegrenzung entstehen bei der Übernahme von voller Budgetverantwortung, wie sie für ein reales Budget typisch ist, stärkere Anreize zur Risikoselektion. Damit wächst auch die Bedeutung der Genauigkeit der Risikoadjustierung. Dies gilt umso mehr, als sich die Verantwortung für ein reales Budget immer nur auf die eingeschriebenen Versicherten beziehen kann. Die Ausweitung der Erfolgsmessung auf alle Versicherten einer kooperierenden Krankenkasse, wie dies im Gesunden Kinzigtal erfolgt, ist nicht möglich.

\section{Interne Budgetverteilung}

Der entscheidende Vorteil bei der Übernahme von Verantwortung für ein reales Budget besteht im Gegensatz zum virtuellen Budget darin, dass die internen Vergütungsstrukturen für die beteiligten Ärzte völlig unabhängig von denen des KV-Systems gestaltet werden können. So wären bei voller realer Budgetverantwortung z.B. ein vergleichsweise höherer Pauschalisierungsgrad und mehr leistungsabhängige Vergütungselemente möglich. Die Ärzte würden zudem verstärkt zu Verhaltensänderungen bei den selbst erbrachten und nicht nur vorrangig bei veranlassten Leistungen angereizt.

Durch die Verantwortung für ein reales Budget gewinnen Modelle der regionalen Vollversorgung an Unabhängigkeit gegenüber der KV. Im Gegensatz zu Einsparverträgen sind sie nicht mehr auf die Abrechnung ihrer Leistungen über das KV-System angewiesen. Sie können daher stärker in Konkurrenz zu den KVen um den Versorgungsauftrag der Krankenkassen treten.

\section{Budgetbereinigung}

Da das Netzwerk das reale Budget tatsächlich als Geldbetrag vor Beginn des Abrechnungszeitraums zur Verfügung gestellt bekommt, wird gegenüber der KV eine Bereinigung der morbiditätsbedingten Gesamtvergütung erforderlich. Ziel der Bereinigung ist es, eine Doppelfinanzierung des Netzwerks durch die beiden Budgets zu vermeiden [27]. Die morbiditätsbedingte
Gesamtvergütung ist daher um den Betrag zu vermindern, den eine Versorgung der Versicherten, die an der integrierten Versorgung teilnehmen, im System der KV gekostet hätte. Bereinigungspflichtig sind Leistungen, die durch das Netzwerk erbracht werden und zugleich auch durch die morbiditätsbedingte Gesamtvergütung der KV abgedeckt werden [2].

Aufgrund der Notwendigkeit einer Budgetbereinigung ergeben sich bei einem realen Budget auch besondere Schwierigkeiten, wenn ein Versicherter Leistungen von Leistungserbringern außerhalb des Netzes in Anspruch nimmt. Diese Leistungen müssen dann aus dem bereits bezahlten Netzbudget finanziert, d.h. zugekauft, werden.

\section{Risikoteilung}

Bei Übernahme von Verantwortung für ein reales Budget werden die Netzärzte voll am Verlustrisiko bei Überschreitung des Budgets beteiligt. Um dieses Risiko zu begrenzen, z. B. gegenüber dem Auftreten von einzelnen aber extrem teuren Krankheitsfällen oder von Epidemien, kommen Rückversicherungslösungen mit den beteiligten Krankenkassen eine gesteigerte Bedeutung zu. Dies ist auch allokativ sinnvoll, da die Krankenkasse über eine wesentlich größere Versichertenpopulation verfügt als das Gesundheitsnetzwerk und deshalb besser in der Lage ist, seltene aber hohe Risiken auszugleichen.

\section{Netzwerkvergütung aus dem Gesundheitsfonds $\nabla$}

Eine konsequente Fortführung der beschriebenen Entwicklung von einem virtuellen Budget hin zur Übernahme realer Budgetverantwortung in der regionalen Vollversorgung läge in der $\mathrm{Zu}-$ weisung eines realen Budget direkt aus dem Gesundheitsfonds, setzt jedoch die Schaffung der notwendigen Rechtsgrundlagen voraus.

\section{Grundlagen und Finanzströme}

Der Gedanke, innerhalb der Vergütungskaskade die Krankenversicherung zu überspringen und eine regionale Vollversorgung direkt aus dem Gesundheitsfonds durch ein reales, aus risikoadjustierten Kopfpauschalen gebildetes Budget zu vergüten, hat 
u.a. die Vergütung von ACOs durch Medicare in den USA zum Vorbild [28]. Ähnliche Möglichkeiten zur direkten Vergütung durch Medicare unter Umgehung zwischengelagerter Versicherungen existieren auch für Integrated Delivery Systems (IDS) [29]. Die entstehenden Strukturen würden in Bezug auf die Risikoteilung aber auch institutionell die Funktionen einer Krankenkasse mit jenen der Leistungserbringung verbinden [28]. Eine Möglichkeit zur Umsetzung einer Finanzierung innovativer Versorgungsformen direkt aus dem Gesundheitsfonds ist in $\bullet$ Abb. 3 dargestellt.

Die Risikoadjustierung des Budgets könnte weiterhin mithilfe des Morbi-RSA erfolgen. Problematischer ist dagegen die Frage des Ausgleichs regionaler Kostenunterschiede, die bisher die Krankenkassen tragen. Solange der Fonds regional undifferenziert bundesweite Durchschnittswerte verteilt, müssten sie für entgangene regionale Vorteile entschädigt werden bzw. ungekehrt bei der Abgabe von Versicherten in Hochkostenregionen eine entsprechende Zahlung an den Fonds leisten.

\section{Vorteile einer direkten Finanzierung aus dem Gesundheitsfonds}

Wie aus der Darstellung ersichtlich, entfällt bei einer direkten Finanzierung der innovativen Versorgungsform aus dem Gesundheitsfonds die Notwendigkeit zur Bereinigung der morbiditätsbedingten Gesamtvergütung bzw. diese kann einfach durch Einbehaltung der entsprechenden Kopfpauschalen erfolgen. So ließe sich eine größere Unabhängigkeit von den Strukturen der Honorarverteilung innerhalb der KVen erreichen.

Eine Finanzierung regionaler Vollversorgungsmodelle direkt aus dem Gesundheitsfonds brächte den Initiativen der Leistungserbringer vor Ort größere Unabhängigkeit von der Investitionsbereitschaft der Krankenkassen. Voraussetzung wäre die zentrale aber subsidiäre Vorgabe eines transparenten Standardmodells zur regionalen Vollversorgung durch den Gesetzgeber. Gründungswillige Leistungserbringer vor Ort könnten dann in jedem Fall das Standardmodell umsetzen.

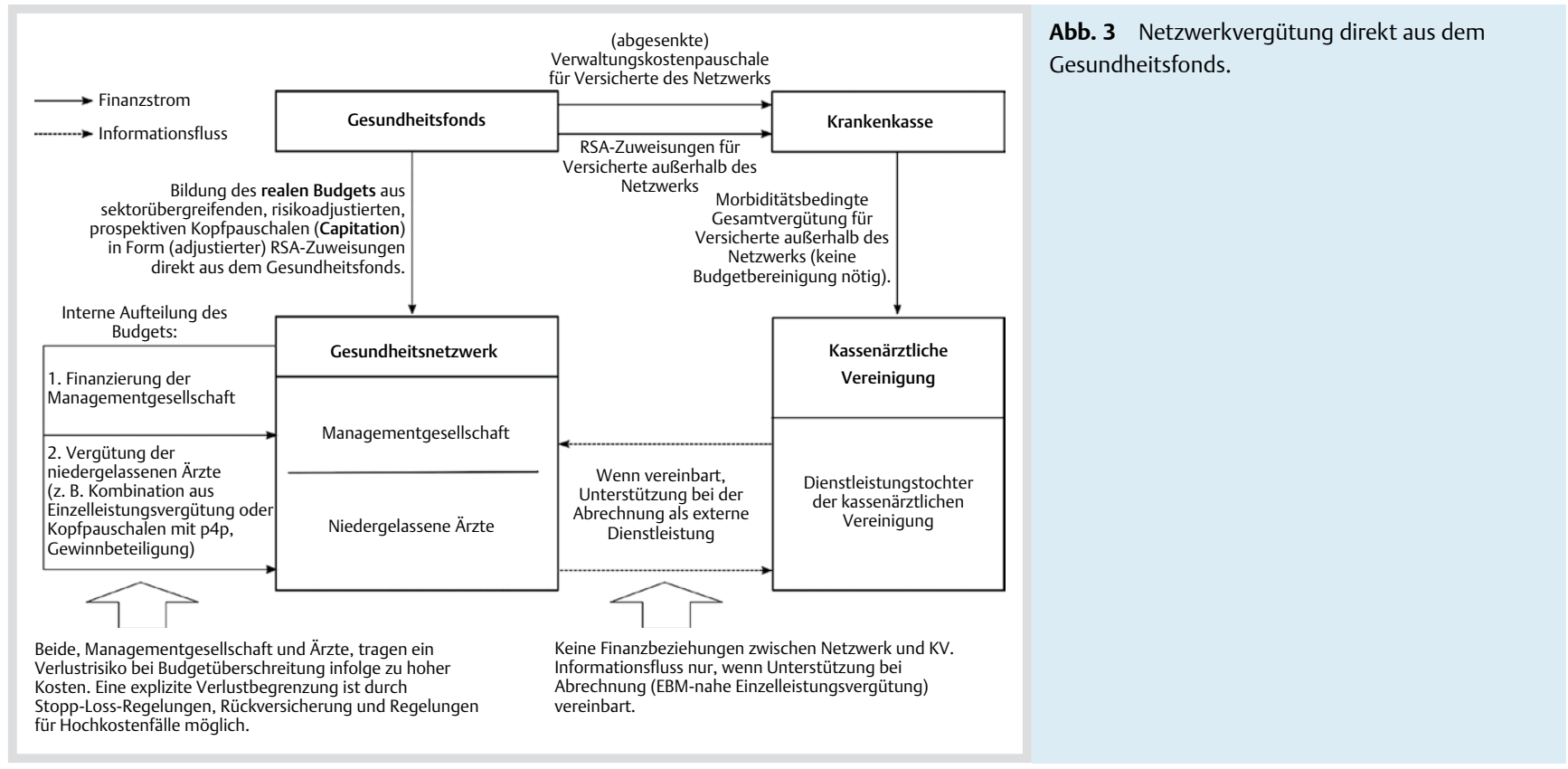

Tab. 3 Chancen und Risiken der diskutierten Vergütungsformen für Gesundheitsnetzwerke.

\begin{tabular}{|c|c|c|}
\hline & Chancen & Risiken \\
\hline $\begin{array}{l}\text { virtuelles } \\
\text { Budget }\end{array}$ & $\begin{array}{l}\text { - Zusatzeinkommen durch Einsparbeteiligungen } \\
\text { - Vermeidung einer Bereinigung des KV-Budgets und damit von } \\
\text { Konflikten } \\
\text { - Einfache Handhabung von Fremdleistungen } \\
\text { - Einstieg in die Übernahme von Budgetverantwortung } \\
\text { - Vermeidung von Selektionsanreizen durch Einbezug von nicht } \\
\text { eingeschriebenen Versicherten möglich }\end{array}$ & $\begin{array}{l}\text { - Konflikte infolge von Einsparungen zu Lasten nicht beteiligter } \\
\text { Leistungserbringer } \\
\text { - bisher nur eingeschränkte (und ggf. intransparente) } \\
\text { Beteiligung am Versicherungsrisiko und daher nur } \\
\text { eingeschränkte Wirtschaftlichkeitsanreize }\end{array}$ \\
\hline $\begin{array}{l}\text { reales } \\
\text { Budget via } \\
\text { Kranken } \\
\text { kasse }\end{array}$ & $\begin{array}{l}\text { - volle Budgetverantwortung (mit Ausnahme transparenter } \\
\text { Rückversicherungs-vereinbarungen) } \\
\text { - umfassende Gestaltungs-möglichkeiten für die interne } \\
\text { Budgetverteilung (z. B. p4p) }\end{array}$ & $\begin{array}{l}\text { - verstärkte Anreize zur Risikoselektion } \\
\text { - Vermeidung von Selektions-anreizen durch Einbezug von } \\
\text { nicht eingeschriebenen Versicherten unmöglich } \\
\text { - Bereinigung des KV-Budgets ist erforderlich und birgt Kon- } \\
\text { fliktpotenzial } \\
\text { - erhöhte Notwendigkeit von Rückversicherungsverträgen für } \\
\text { seltene Hochkostenfälle }\end{array}$ \\
\hline $\begin{array}{l}\text { reales } \\
\text { Budget via } \\
\text { Gesund } \\
\text { heitsfonds }\end{array}$ & $\begin{array}{l}\text { - Wegfall der Notwendigkeit einer Budgetbereinigung } \\
\text { - Unabhängigkeit von der Investitionsbereitschaft der Krankenkassen } \\
\text { - auch institutionelle Integration von Versicherung und } \\
\text { Leistungserbringung }\end{array}$ & $\begin{array}{l}\text { - zu schaffende rechtliche Voraussetzungen } \\
\text { - erhöhte Managementaufgaben durch die auch institutionell } \\
\text { voll integrierte Versicherungsfunktion }\end{array}$ \\
\hline
\end{tabular}


Ähnlich wie bei den ACOs und den Medicare Advantage Plans sollten sich die Vorgaben des Standardmodells auf die Vergütung unter Einbeziehung der Qualitätssicherung beschränken. Die interne Organisation der Versorgung sollte dagegen den Netzen überlassen bleiben, um die versorgungsnahe Entwicklung von Strukturen „vor Ort“ zu ermöglichen. Voraussetzung wäre die Entwicklung konsensfähiger, wissenschaftlich fundierter Instrumente zur Qualitätsmessung und -bewertung. Hier läge ein wichtiges Feld für die Versorgungsforschung.

\section{Diskussion und Fazit}

$\nabla$

- Tab. 3 fasst die Chancen und Risiken der dargestellten Vergütungsformen für Gesundheitsnetzwerke zusammen. Veröffentlichte Evaluationsergebnisse liegen bisher nur für Gesundheitsnetzwerke mit virtuellem Budget vor. Effekte der neuartigen Übernahme realer Budgetverantwortung durch das UGOM wurden bisher nicht publiziert, sodass für eine vergleichende Bewertung vorerst auf die diskutierten Vor- und Nachteile sowie internationale Erfahrungen mit vergleichbaren Modellen zurückgegriffen werden muss. Für das Zukunftsmodell einer pauschalen Vergütung direkt aus dem Gesundheitsfonds gilt dies in besonderem Maße.

Zusammenfassend lässt sich feststellen, dass jede der 3 skizzierten Vergütungsstrukturen spezifische Chancen und Risiken birgt. Betrachtet man Netzwerke dynamisch im Lebenszyklus, können virtuelle Budgets aufgrund der eingeschränkten Risikobeteiligung als Einstieg in die Übernahme von Budgetverantwortung gesehen werden. Reifere Netze können dagegen auch reale Budgetverantwortung übernehmen und so weitere Wirtschaftlichkeitspotenziale heben. Die Verwaltung eines Budgets, das direkt aus dem Gesundheitsfonds zugwiesen wird, stellt wegen der damit verbundenen vollständigen Integration von Versicherung und Leistungserstellung die größte Herausforderung dar, böte den Netzen aber andererseits auch die größte Unabhängigkeit. Die Ausdifferenzierung und Weiterentwicklung der diskutierten Gestaltungsoptionen für die Vergütung von Gesundheitsnetzwerken wird auch künftig ein wichtiges Gebiet der netzwerkorientierten Versorgungsforschung bilden.

Interessenkonflikt: Die Autoren geben an, dass kein Interessenkonflikt besteht

\section{Literatur}

1 SVR-Gesundheit. Gutachten 2009 des Sachverständigenrates zur Begutachtung der Entwicklung im Gesundheitswesen. Koordination und Integration - Gesundheitsversorgung in einer Gesellschaft des längeren Lebens; 2009

2 SVR-Gesundheit. Wettbewerb an der Schnittstelle zwischen ambulanter und stationärer Gesundheitsversorgung. BT-Drs. 17/10323 2012

3 Braun GE, Güssow J, Heßbrügge G et al., Hrsg. Innovative Versorgungsformen im Gesundheitswesen. Konzepte und Praxisbeispiele erfolgreicher Finanzierung und Vergütung. Köln: Deutscher Ärzte-Verlag, 2009

4 Schwartz FW, Arbeitskreis Versorgungsforschung beim Wissenschaftlichen Beirat der Bundesärztekammer. Definition und Abgrenzung der Versorgungsforschung 2004

5 Pfaff $H$. Versorgungsforschung. Begriffsbestimmung, Gegenstand und Aufgaben. In: Pfaff H, Schrappe M, Lauterbach KW et al., Hrsg. Gesundheitsversorgung und Disease Management. Grundlagen und Anwendungen der Versorgungsforschung. 1. Aufl. Bern [u.a.]: Huber, 2003; 13-23

6 Schöffski O. Grundformen gesundheitsökonomischer Evaluationen. In: Schöffski O, von der Schulenburg JG, Hrsg. Gesundheitsökonomische Evaluationen. 3. Aufl. Berlin: Springer, 2008

7 Pfaff $H$, Albert $U$, Bornemann R. Methoden für die organisationsbezogene Versorgungsforschung. Das gesundheitswesen 2009; 777-790
8 Sydow J. Strategische Netzwerke. Evolution und Organisation. Wiesbaden: Gabler, 1992

9 Braun GE, Güssow J. Integrierte Versorgungsstrukturen und Gesundheitsnetzwerke als innovative Ansätze im deutschen Gesundheitswesen. In: Braun GE, Schulz-Nieswandt F, Hrsg. Liberalisierung im Gesundheitswesen. Einrichtungen des Gesundheitswesens zwischen Wettbewerb und Regulierung. 1. Aufl. Baden-Baden: Nomos, 2006; 65-93

10 Schreyögg $J$, Weinbrenner $S$, Busse R. Leistungsmanagement in der Integrierten Versorgung. In: Busse R, Schreyögg J, Tiemann O, Hrsg. Management im Gesundheitswesen. 2. Aufl. Heidelberg: Springer, 2010; 101-121

11 Braun GE, Schumann A, Güssow J. Bedeutung innovativer Versorgungsformen und grundlegende Finanzierungs- und Vergütungsaspekte: Einführung und Überblick über die Beiträge. In: Braun GE, Güssow J, Heßbrügge G et al., Hrsg. Innovative Versorgungsformen im Gesundheitswesen. Konzepte und Praxisbeispiele erfolgreicher Finanzierung und Vergütung. Köln: Deutscher Ärzte-Verlag, 2009; 3-20

12 Braun GE, Binder A, Burkart J et al. Managed Care für Deutschland - Ein ganzheitliches Konzept unter besonderer Berücksichtigung von Finanzierungsüberlegungen. In: Porzsolt F, Hrsg. Managed Care. Perspektive für das deutsche Gesundheitswesen? 1. Aufl. Berlin: PVS, 2010; 104-128

13 Hildebrandt H, Bischoff-Everding $C$, Saade $P$ et al. Finanzierung und Vergütung der Integrierten Versorgung aus Sicht einer Managementgesellschaft - das Beispiel Gesundes Kinzigtal. In: Braun GE, Güssow J, Heßbrügge $G$ et al., Hrsg. Innovative Versorgungsformen im Gesundheitswesen. Konzepte und Praxisbeispiele erfolgreicher Finanzierung und Vergütung. Köln: Deutscher Ärzte-Verlag, 2009; 49-64

14 Bahr TM. Finanzierung und Vergütung aus Sicht eines Modells der populationsbezogenen regionalen Vollversorgung - am Beispiel des Unternehmens Gesundheit Oberpfalz Mitte Gmb |\& Co KG (UGOM). In: Braun GE, Güssow J, Heßbrügge $G$ et al., Hrsg. Innovative Versorgungsformen im Gesundheitswesen. Konzepte und Praxisbeispiele erfolgreicher Finanzierung und Vergütung. Köln: Deutscher Ärzte-Verlag, 2009; 65-73

15 Wambach $V$, Lindenthal J. Finanzierung und Vergütung aus Sicht der Ärztegenossenschaft Qualität und Effizienz - QuE Nürnberg. In: Braun GE, Güssow J, Heßbrügge G et al., Hrsg. Innovative Versorgungsformen im Gesundheitswesen. Konzepte und Praxisbeispiele erfolgreicher Finanzierung und Vergütung. Köln: Deutscher Ärzte-Verlag 2009; 39-48

16 Daul G. Das Projekt „Gesundes Kinzigtal“.In: AOK-Bundesverband., Hrsg. Wettbewerb für Patienten. Mit selektiven Verträgen Versorgungsqualität gestalten, Berlin: 2008; 33-43

17 Hildebrandt $H$, Hermann C, Knittel $R$ et al. Gesundes Kinzigtal Integrated Care: improving population health by a shared health gain approach and a shared savings contract. Int Integr Care 2010; 10: 1-15

18 Green L. Evaluation of CMMI Accountable Care Organization Initiatives. Effect of Pioneer ACOs on Medicare Spending in the First Year 2013;

19 Schulenburg JG. Gesundheitsökonomik. 2. Aufl. Tübingen: Mohr Siebeck, 2007

20 Social Security Administration. Medicare Premiums: Rules For Higher-Income Beneficiaries.Im Internet http://www.ssa.gov/pubs/EN-0510536.pdf Stand: 11.06 .2013

21 Department of Health and Human Services, Centers for Medicare and Medicaid Services. Medicare Program; Medicare Shared Savings Program: Accountable Care Medicare Programm - Medicare Shared Savings Programm: Accountable Care Organizations; Final Rule. Fed Regist 2011; 76: 67802-67990

22 UGOM. Die UGOM Erfolgsgeschichte.Im Internet http://www.ugom. de/Historie.93.0.html Stand: 02.08.2013

23 Medicare Payment Advisory Commission Medpac. Medicare Payment Policy. Report to the Congress 2013;

24 Medpac. Medicare Advantage Programm - payment system. Washington DC: 2012

25 Dieguez G, Piper B, Clark A. The Medicare Advantage 5-Star Rating Program and Its Implications for Actuaries. Health Watch 2012; 1-9

26 Jäger C, Cornelius F, Braun GE. Wirtschaftliche Kalkulation und Zukunft großer Medizinischer Versorgungszentren (MVZ). In: Braun GE, Güssow J, Heßbrügge $G$ et al., Hrsg. Innovative Versorgungsformen im Gesundheitswesen. Konzepte und Praxisbeispiele erfolgreicher Finanzierung und Vergütung. Köln: Deutscher Ärzte-Verlag, 2009; 147-161

27 Wasem J. Wie sich Versorgungsqualität optimieren lässt. In: AOK-Bundesverband., Hrsg. Wettbewerb für Patienten. Mit selektiven Verträgen Versorgungsqualität gestalten. Berlin: 2008; 63-72

28 NCSL. Accountable Care Organizations. Denver Colorado 2010

29 Amelung VE. Managed Care. Neue Wege im Gesundheitsmanagement. 5. Aufl. Wiesbaden: Betriebswirtschaftlicher Verlag Gabler, 2012 